Pacific Journal of Mathematics

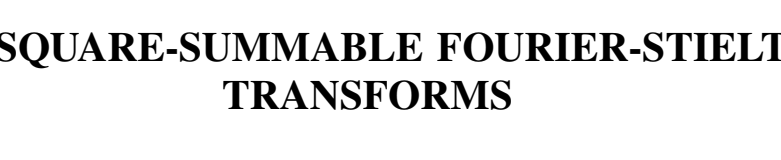




\section{SEMI-SQUARE-SUMMABLE FOURIER-STIELTJES TRANSFORMS}

\section{GLICKSBERG}

For $G$ a locally compact abelian group with dual $\Gamma$, let $\mu$ be a (finite regular Borel) measure on $G$ with Fourier-Stieltjes transform $\hat{\mu}$. Doss has recently shown that when $\Gamma$ is (algebraically) a totally ordered abelian group and $\hat{\mu}$ is square integrable on the negative half $\Gamma_{-}$of $\Gamma$ then its singular component $\sigma$ has $\hat{\sigma}=0$ on $\Gamma_{-}$; in particular $\mu E=0$ for each common null set $E$ of the analytic measures (those with transforms 0 on $\Gamma_{-}$), such $E$ being Haar-null.

In the similar (but usually distinct) case in which $\Gamma$ is partially ordered by a nonzero homomorphism $\psi: \Gamma \rightarrow R$ with $\Gamma_{-}=\psi^{-1}(-\infty, 0]$ the common null sets $E$ are known, and our purpose is to note in this setting how function algebra results apply to show $\mu E=0$ when $\hat{\mu} \in L^{2}\left(\Gamma_{-}\right)$, and when $\hat{\mu}$ satisfies sometimes weaker (but more obscure) hypotheses.

Doss' results appear in [2], and the function algebra results we apply are those in $[4, \S 1],[5, \S 2]$, with which we shall assume the reader familiar. The common null sets mentioned above are given in $[1,5]$.

Theorem 1. Let $\psi, \Gamma_{-}$be as above and let $\varphi: R \rightarrow G$ be the homomorphism dual to $\psi$. If

$$
\int_{\Gamma_{-}}|\hat{\mu}(\gamma)|^{2} d \gamma<\infty
$$

then $\mu$ vanishes on all Borel $E \subset G$ for which

$$
\{t \in R: x+\varphi(t) \in E\} \text { has linear measure } 0,
$$

for all $x \in G$, i.e. (by definition [3, §2]) $\mu$ is absolutely continuous in the direction of $\varphi$.

Proof. Let $G^{a}$ be the Bohr compactification of $G$, with dual $\Gamma_{d}$, the discrete version of $\Gamma$. Dual to $\psi: \Gamma_{d} \rightarrow R$ we have a map of $R$ into $G^{a}$, the composition $R \stackrel{\varphi}{\longrightarrow} G \rightarrow G^{a}$, which we still call $\varphi$. Note that each Borel $E$ in $G$ is Borel ${ }^{1}$ in $G^{a}$, and if $E \subset G$ satisfies (2) for $x \in G$ it does for all $x$ in $G^{a}$ (the set is void for $x \in G^{a} \backslash G$ ). As in [5] we are forced to transfer our attention to $G^{a}$ to apply the function algebra results.

\footnotetext{
1 We take the $\sigma$-ring generated by compacta as our Borel sets.
} 
Let $A$ be the closed span of $\Gamma_{+}=\psi^{-1}[0, \infty)$ in $C\left(G^{a}\right)$, a subalgebra of $C\left(G^{a}\right)$. As usual we can shift $\mu$ to a measure on $G^{a}$ carried by its subset $G$ [6] with the same Fourier-Stieltjes transform as before. Let $\hat{f}$ be the element $\hat{\mu} \chi$ of $L^{2}(\Gamma)$, where $\chi$ is the characteristic function of $\Gamma_{-}$, and ${ }^{2} f$ the element of $L^{2}(G)$ corresponding to $\hat{f}$.

For any trigonometric polynomial $p=\sum c_{i} \gamma_{i}$ in $A$ (i.e., with $\left.\psi^{\prime}\left(\gamma_{i}\right) \geqq 0\right)$ we have

$$
(p \mu)^{\wedge}(\gamma)=\int \bar{\gamma} p d \mu=\sum c_{i} \hat{\mu}\left(\gamma-\gamma_{i}\right)=\left(\sum c_{i} \delta_{-\gamma_{i}}\right) * \hat{\mu}(\gamma),
$$

and since

$$
\begin{aligned}
\left(\sum c_{i} \delta_{-\gamma_{i}}\right) * \hat{f}(\gamma) & =\sum c_{i} \hat{\mu}\left(\gamma-\gamma_{i}\right) \chi\left(\gamma-\gamma_{i}\right) \\
& =\sum c_{i} \hat{\mu}\left(\gamma-\gamma_{i}\right)=(p \mu)^{\wedge}(\gamma)
\end{aligned}
$$

if $\psi(\gamma) \leqq 0$, we have

$$
\int_{\Gamma_{-}}\left|(p \mu)^{\wedge}\right|^{2} d \gamma \leqq \int_{\Gamma}\left|\left(\sum c_{i} \delta_{-\gamma_{i}}\right) * \widehat{f}\right|^{2} d \gamma=\int_{G}|p|^{2} d x \leqq\|p\|_{\infty}^{2}\|f\|_{2}^{2}
$$

or

$$
\left\|(p \mu)^{\wedge} \chi\right\|_{2} \leqq\|f\|_{2} \cdot\|p\|_{\infty} \text {. }
$$

Now (3) continues to hold for any $a \in A$ in place of $p \in A$ : for if $p_{n} \rightarrow a$ in $A$ then $\left(p_{n} \mu\right)^{\wedge} \rightarrow(a \mu)^{\wedge}$ uniformly, so that for any compact $K \subset \Gamma_{-}$

$$
\begin{aligned}
\int_{K}\left|(a \mu)^{\wedge}\right|^{2} d \gamma & =\lim \int_{K}\left|\left(p_{n} \mu\right)^{\wedge}\right|^{2} d \gamma \leqq\|f\|_{2}^{2} \lim \left\|p_{n}\right\|_{\infty}^{2} \\
& =\|f\|_{2}^{2}\|a\|_{\infty}^{2}
\end{aligned}
$$

whence $\left\|(a \mu)^{\wedge} \chi\right\|_{2} \leqq\|f\|_{2}\|a\|_{\infty}$. Indeed this clearly follows whenever $\left\|p_{n}\right\|_{\infty} \leqq\|a\|_{\infty}$ and $\left(p_{n} \mu\right)^{\wedge} \rightarrow(a \mu)^{\wedge}$ uniformly.

Let $\gamma$ be a fixed element of $\Gamma$ with $\psi(\gamma)>0$, and let $\mu=\nu+\sigma$ be the Lebesgue decomposition of $\mu$ relative to $M^{r}$ (the probability measures on $G^{a}$ orthogonal to $\gamma A$, ef. [4, §1]), with $\nu \ll M^{\gamma}, \sigma M^{\gamma_{-}}$ singular. By the argument of the last paragraph of $[5, \S 2], \nu$ vanishes on Borel sets in $G^{a}$ satisfying (2), so we can complete our proof by showing $\sigma=0$. As in [4] $\sigma$ is carried by $\cup K_{n}$, where $K_{n}$ is a compact $M^{\gamma}$-null set.

By the abstract Forelli Lemma [4, 1.2] (applied to the algebra C $+\gamma A$ ) and dominated convergence we have $\left\{a_{n}\right\}$ in the unit ball of $A$ for which $a_{n} \mu \rightarrow \sigma$ in norm, so $\left(a_{n} a \mu\right)^{\wedge} \rightarrow(a \sigma)^{\wedge}$ uniformly and again we conclude that $\left\|(a \sigma)^{\wedge} \chi\right\|_{2} \leqq\|f\|_{2}\|a\|_{\infty}$ for $a \in A$.

${ }^{2}$ It should be noted that when $G$ is compact $f \in L^{1}(G)$ and the result follows trivially from [1]; for then $\nu(d x)=\mu(d x)-f(x) d x$ defines an analytic measure. 
Now by [5, §2] each measure $\tau$ on $G^{a}$ orthogonal to $A$ has $\tau_{K_{n}}=0$ for each $K_{n}$ and thus by [3, 4.8] $K_{n}$ is an intersection of peak sets of $A$, and an interpolation set for $A$; using the regularity of $\sigma$ one then concludes ${ }^{3}$ there is a sequence $\left\{a_{j}\right\}$ in the unit ball of $A$ for which $a_{j} \sigma \rightarrow\left|\sigma_{K_{n}}\right|$ in norm. So again $\left\|\left|\sigma_{K_{n}}\right|^{\wedge} \cdot \chi\right\|_{2} \leqq\|f\|_{2} \cdot 1$, which of course implies $\left|\sigma_{K_{n}}\right|^{\wedge} \in L^{2}(\Gamma)$ since the absolute value of this function is even. Because $\mu$ is carried by the subset $G$ of $G^{a}$, the same is true of its restrictions $\sigma$ and $\sigma_{K_{n}}$ and so, as a measure on $G$ with square summable transform, $\left|\sigma_{K_{n}}\right|$ is absolutely continuous by the elementary argument given by Doss [2, Th. 1]. Hence $\sigma$ is absolutely continuous.

To complete our proof we can show $\sigma=0$ by showing $\sigma$ is carried by a Haar-null set. And since $\sigma$ is carried by a $\sigma$-compact set, it suffices to show $\sigma_{x_{0}+V}$ is carried by a Haar null set for each $x_{0} \in G$ and some compact symmetric neighborhood $V$ of the identity. But $\sigma$ and each $\lambda \in M^{\gamma}$ are mutually singular, so it suffices to show there is a $\lambda$ in $M^{\gamma}$ equivalent to Haar measure on $x_{0}+V$, and, for example, with $m$ Haar measure

$$
\lambda E=\int_{-\infty}^{\infty} \int_{E} \frac{1}{m 2 V} \chi_{x_{0}+2 V}(x-\varphi(t)) d x \rho(t) d t
$$

defines such a measure if

$$
\hat{\rho}(s)=\left\{\begin{array}{l}
1-\frac{|s|}{\psi(\gamma)},|s| \leqq \psi(\gamma) \\
0 \text { elsewhere }
\end{array}\right.
$$

Indeed

$$
\rho(t)=\psi(\gamma)\left(\frac{\sin t \psi(\gamma) / 2}{t / 2}\right)^{2} \geqq 0
$$

so $\lambda \geqq 0$ and

$$
\hat{\lambda}(\gamma)=\frac{1}{m 2 V} \hat{\chi}_{x_{0}+2 V}(\gamma) \cdot \hat{\rho}(\psi(\gamma)),
$$

as is easily verified; so $\hat{\lambda}$ vanishes off $\psi^{-1}(-\psi(\gamma), \psi(\gamma))$ whence $\lambda$ is orthogonal to $\gamma A$, the span of $\{\beta \in \Gamma: \psi(\beta) \geqq \psi(\gamma)\}$. And $\lambda E=0$ implies

$$
\int_{E} \chi_{x_{0}+2 V}\left(x-\varphi\left(t_{0}\right)\right) d x=0
$$

for some $t_{0}$ with $\varphi\left(t_{0}\right) \in V$ since $\rho(t)>0$ a.e., $\varphi(0) \in V$ and $\varphi$ is con-

3 By regularity there is a peak set (an intersection of countably many such) $F_{n} \supset K_{n}$ for which $\sigma_{F_{n}}=\sigma_{K_{n}}$, and if $f$ peaks on $F_{n}$ then $f^{k} \rightarrow 1$ a.e. $\left|\sigma_{K_{n}}\right|, \rightarrow 0$ a.e. $\left|\sigma_{K_{n}^{\prime}}\right|$. If $\sigma_{K_{n}}=\rho\left|\sigma_{K_{n}}\right|,|\rho| \equiv 1$, then we have $f_{k}$ in the unit ball of $C\left(K_{n}\right)^{n}$ for which $f_{k} \rightarrow \rho$ a.e. $\left|\sigma_{K_{n}}\right|$, hence $b_{k}$ in the $(1+\varepsilon)$-ball of $A$ for which $b_{k}=f_{k}$ on $K_{n}$, whence $b_{k} f^{k} \sigma \rightarrow\left|\sigma_{K_{n}}\right|$ by dominated convergence. 
tinuous, so if $E \subset x_{0}+V$ we have $E-\varphi\left(t_{0}\right) \subset x_{0}+2 V$, and therefore

$$
0=\int_{E} \chi_{x_{0}+2 v}\left(x-\varphi\left(t_{0}\right)\right) d x=\int_{E} 1 d x=m E .
$$

Hence $m_{x_{0}+2 V} \ll \lambda_{x_{0}+2 V}$; the reverse is obvious (and actually unnecessary) and our proof complete.

Variants of theorem 1 can be obtained from the same argument, but seem to require more artificial hypotheses. For example

THEOREM 2. With if, $\Gamma_{-}$as before, suppose the continuous function $f=f^{*} \in L^{1}(G) \cap L^{1}(\Gamma)^{\wedge}$ never vanishes on $n^{4} G$, and $\mu$ is a measure for which for some $k$

$$
\int_{I}\left|\int_{I_{-}}(p \mu)^{\wedge}(\gamma) \hat{f}(\beta-\gamma) d \gamma\right|^{2} d \beta \leqq k\|p\|_{\infty}
$$

for all trigonometric polynomials $p=\sum_{i=1}^{n} c_{i} \gamma_{i}$ with $\psi\left(\gamma_{i}\right) \geqq 0$. Then $\because E=0$ for each Borel $E \subset G$ satisfing (2).

We argue exactly as before that if $p_{n} \mu \rightarrow a \mu$ and $\left\|p_{n}\right\|_{\infty} \leqq\|a\|_{\infty}$, $a \in A$, one has

$$
\int_{K} \mid \int_{\Gamma_{-}}\left(\left.a \iota^{\wedge} \wedge(\gamma) \hat{f}(\beta-\gamma) d \gamma\right|^{2} d \beta \leqq k\|a\|_{\infty}\right.
$$

for $K$ compact, so (4) holds for $p$ an arbitrary element of $A$.

With $\mu=\nu+\sigma$ as before we again obtain (4) for $p \in A$ and $\sigma$ in place of $\mu$, and then for $1=p \in A$ and $\left|\sigma_{K_{n}}\right|=\tau$ in place of $\sigma$. But since $\overline{\hat{\tau}}(-\gamma)=\hat{\tau}(\gamma)$ the finite integral

$$
\int_{\Gamma^{\prime}}\left|\int_{r_{-}} \hat{\tau}(\gamma) \hat{f}(\beta-\gamma) d \gamma\right|^{2} d \beta
$$

coincides with

$$
\begin{aligned}
\int_{\Gamma}\left|\int_{\Gamma_{-}} \overline{\hat{\tau}}(-\gamma) \hat{f}(\beta-\gamma) d \gamma\right|^{2} d \beta & =\int_{\Gamma}\left|\int_{\Gamma_{-}} \overline{\hat{\tau}}(-\gamma) \overline{\hat{f}}(\gamma-\beta) d \gamma\right|^{2} d \beta \\
& =\int_{\Gamma}\left|\int_{\Gamma_{+}} \hat{\tau}(\gamma) \hat{f}(-\gamma-\beta) d \gamma\right|^{2} d \beta \\
& =\int_{\Gamma}\left|\int_{\Gamma_{+}} \hat{\tau}(\gamma) \hat{f}(\beta-\gamma) d \gamma\right|^{2} d \beta
\end{aligned}
$$

so that, by Minkowski, $\hat{\tau} * \hat{f} \in L^{2}(\Gamma)$. Trivially one verifies that the transform of the finite measure $f \tau$, on $G$ is $\hat{\tau} * \widehat{f}$ : thus $f \tau$ is absolutely

${ }^{4}$ When such an $f$ exists this contains the preceding result. For when $\hat{\mu} \chi \in L^{2}(\Gamma)$ so is $(p \mu)^{\wedge} \chi$ and always of norm $\leqq k\|p\|_{\infty}$ as we saw in the proof of Theorem 1 . But then $\left\|(p \mu)^{\wedge} \chi * \hat{f}\right\|_{2} \leqq\left\|(p \mu)^{\wedge} \chi\right\|_{2}\|\hat{f}\|_{1} \leqq k\|p\|_{\infty}\|\hat{f}\|_{1}$ which is (4). 
continuous, so $\tau=\left|\sigma_{K_{n}}\right|$ is since $f$ never vanishes; again $\sigma$ is singular with respect to Haar measure, and $\sigma=0$ follows.

THeorem 3. Suppose there are $\gamma_{n} \in \Gamma$ for which $\varepsilon_{n}=\left\|\bar{\gamma}_{n} \mu\right\|_{A^{*}} \rightarrow 0$, where the norm is that of $\bar{\gamma}_{n} \mu$ as a functional on $A=\operatorname{span} \Gamma_{+}$. Then $\mu E=0$ for every Borel $E$ in $G$ satisfying (2).

We are supposing that $\left|(\alpha \mu)^{\wedge}\left(\gamma_{n}\right)\right| \leqq \varepsilon_{n}\|a\|_{\infty}$ for each $a \in A$, where $\varepsilon_{n} \rightarrow 0$. As before we have $a_{j} \in A,\left\|a_{j}\right\| \leqq 1$, with $a_{j} \mu \rightarrow \sigma$, where $\sigma$ is the $M^{r}$-singular component of $\mu$, so

$$
\left|(\alpha \sigma)^{\wedge}\left(\gamma_{n}\right)\right| \leqq \varepsilon_{n}\|a\|_{\infty}
$$

follows since $\left(\alpha_{j} \cdot a \mu\right)^{\wedge} \rightarrow(\alpha \sigma)^{\wedge}$ uniformly. Now we have $\sigma$ carried by $\cup K_{j}, K_{j}$ a compact $M^{\gamma}$-null set, and as before an intersection of peak sets of $A$ and an interpolation set for $A$. So exactly as before (cf. footnote 3) we have $\left\{a_{k}\right\}$ in the unit ball of $A$ for which $a_{k} \sigma \rightarrow \gamma_{n}\left|\sigma_{K_{j}}\right|$, whence by (7)

$$
\left|\left(\gamma_{n}\left|\sigma_{K_{j}}\right|\right)^{\wedge}\left(\gamma_{n}\right)\right|=\left|\sigma_{K_{j}}\right|(1)=\left\|\sigma_{K_{j}}\right\| \leqq \varepsilon_{n}
$$

for all $n$, so $\sigma_{K_{j}}=0, \sigma=0$, completing our proof as before.

As a final remark, we note that for any measure $\mu$ vanishing on all $E$ satisfying (2), i.e., for $\mu$ absolutely continuous in the direction of $\varphi$, if $\left|\psi\left(\gamma_{n}\right)\right| \rightarrow \infty$, we (at least) have $\bar{\gamma}_{n} \mu \rightarrow 0$ weakly..$^{5}$ Indeed since $\Gamma \mu=\{\gamma \mu: \gamma \in \Gamma\}$ is conditionally weakly compact we need only see any weak cluster point of $\left\{\bar{\gamma}_{n} \mu\right\}$ must be 0 , so it suffices to show

$$
\left(\bar{\gamma}_{n} \mu\right)^{\wedge}(\gamma)=\hat{\mu}\left(\gamma+\gamma_{n}\right) \rightarrow 0 \text {. }
$$

But this follows directly from the following easy "Riemann-Lebesgue lemma": If $\mu$ is absolutely continuous in the direction of $\phi$ then for any $\varepsilon>0$ there is an $N$ for which $|\hat{\mu}(\gamma)|<\varepsilon$ if $|\psi(\gamma)|>N$.

By $[3,2.4] \mu$ translates continuously in the direction of $\varphi$, i.e., $\left\|\mu-\mu_{t}\right\|<\varepsilon$ if $|t|<\delta$, where $\mu_{t} E=\mu(\varphi(t)+E)$. Thus for an appropriate continuous $f$ on $R$ vanishing off $(-\delta, \delta)$ we have

$$
\|\mu * f-\mu\|<\varepsilon \text {, }
$$

where

$$
\mu * f=\int \mu_{t} f(t) d t
$$

${ }^{5}$ Thus for any measure $\mu$ on $G$ one has an analogue of a well known lemma of Helson: if $\left|\psi\left(\gamma_{n}\right)\right| \rightarrow \infty$, any weak cluster point $\nu$ of $\left\{\gamma_{n} \mu\right\}$ is carried by a subset $E$ of $G$ satisfying (2), i.e., null in the direction of $\varphi$ in the terminology of [3]. (For $y$ is necessarily a weak cluster point of $\left\{\gamma_{n} \sigma\right\}$, where $\sigma$ is the $M^{\gamma}$-singular component of $\mu$, as always.) 
can be interpreted as, say, a Riemann integral. But

$$
\begin{aligned}
& (\mu * f)^{\wedge}(\gamma)=\iint \overline{(x, \gamma)} \mu_{t}(d x) f(t) d t \\
& =\iint \overline{(x-\varphi(t), \gamma)} \mu(d x) f(t) d t \\
& =\hat{\mu}(\gamma) \int(\varphi(t), \gamma) f(t) d t \\
& =\widehat{\mu}(\gamma) \int(t, \psi(\gamma)) f(t) d t=\hat{\mu}(\gamma) \hat{f}(-\psi(\gamma))
\end{aligned}
$$

which shows $(\mu * f)^{\wedge}$ has the desired property by the Riemann-Lebesgue lemma applied to $f$. As a uniform limit of such functions $\hat{\mu}$ of course has the same property.

\section{REFERENCES}

1. K. deLeeuw and I. Glicksberg, Quasi-invariance and analyticity of measures on compact groups, Acta Math. 109 (1963) 179-205.

2. R. Doss, On measures with small transforms, Pacific J. Math. 26 (1968), 257-263.

3. I. Glicksberg, Measures orthogonal to algebras and sets of antisymmetry, Trans. Amer. Math. Soc. 105 (1962), 415-435.

4. - The abstract F. and M. Riesz theorem, J. Funct. Anal. 1 (1967), 109-122.

5. - Extensions of the F. and M. Riesz theorem, J. Funct. Anal. (to appear).

6. W. Rudin, Fourier analysis on groups, Interscience, New York, 1962.

Received December 23, 1968. Work supported in part by the National Science Foundation.

UNIVERSITY OF WASHINGTON 


\section{PACIFIC JOURNAL OF MATHEMATICS}

\section{EDITORS}

\author{
H. ROYDEN \\ Stanford University \\ Stanford, California \\ Richard Pierce \\ University of Washington \\ Seattle, Washington 98105
}

\author{
J. DugundJI \\ Department of Mathematics \\ University of Southern California \\ Los Angeles, California 90007 \\ BASIL GoRDON \\ University of California \\ Los Angeles, California 90024
}

\section{ASSOCIATE EDITORS}
E. F. BECKENBACH
B. H. Neumann
F. WOLF
K. YoSHIDA

\section{SUPPORTING INSTITUTIONS}

\author{
UNIVERSITY OF BRITISH COLUMBIA \\ CALIFORNIA INSTITUTE OF TECHNOLOGY \\ UNIVERSITY OF CALIFORNIA \\ MONTANA STATE UNIVERSITY \\ UNIVERSITY OF NEVADA \\ NEW MEXICO STATE UNIVERSITY \\ OREGON STATE UNIVERSITY \\ UNIVERSITY OF OREGON \\ OSAKA UNIVERSITY \\ UNIVERSITY OF SOUTHERN CALIFORNIA
}

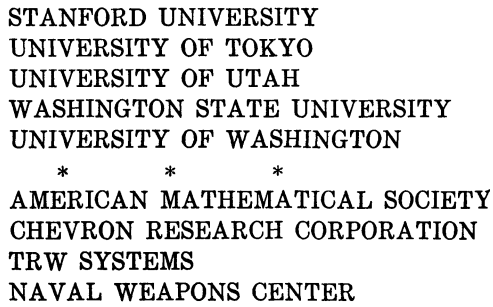

The Supporting Institutions listed above contribute to the cost of publication of this Journal, but they are not owners or publishers and have no responsibility for its content or policies.

Mathematical papers intended for publication in the Pacific Journal of Mathematics should be in typed form or offset-reproduced, double spaced with large margins. Underline Greek letters in red, German in green, and script in blue. The first paragraph or two must be capable of being used separately as a synopsis of the entire paper. It should not contain references to the bibliography. Manuscripts, in duplicate if possible, may be sent to any one of the four editors. Please classify according to the scheme of Math. Rev. 36, 1539-1546. All other communications to the editors should be addressed to the managing editor, Richard Arens, University of California, Los Angeles, California, 90024.

50 reprints are provided free for each article; additional copies may be obtained at cost in multiples of 50 .

The Pacific Journal of Mathematics is published monthly. Effective with Volume 16 the price per volume (3 numbers) is $\$ 8.00$; single issues, $\$ 3.00$. Special price for current issues to individual faculty members of supporting institutions and to individual members of the American Mathematical Society: $\$ 4.00$ per volume; single issues $\$ 1.50$. Back numbers are available.

Subscriptions, orders for back numbers, and changes of address should be sent to Pacific Journal of Mathematics, 103 Highland Boulevard, Berkeley, California, 94708.

PUBLISHED BY PACIFIC JOURNAL OF MATHEMATICS, A NON-PROFIT CORPORATION

Printed at Kokusai Bunken Insatsusha (International Academic Printing Co., Ltd.), 7-17, Fujimi 2-chome, Chiyoda-ku, Tokyo, Japan. 


\section{Pacific Journal of Mathematics}

\section{Vol. 31, No. $2 \quad$ December, 1969}

Efraim Pacillas Armendariz, Quasi-injective modules and stable torsion

classes..........................................

J. Adrian (John) Bondy, On Ulam's conjecture for separable graphs...

Vasily Cateforis and Francis Louis Sandomierski, On commutative rings over which the singular submodule is a direct summand for every module .....

Rafael Van Severen Chacon, Approximation of transformations with continuous

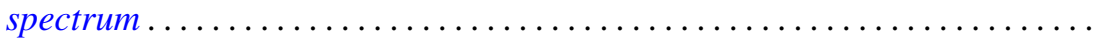

Raymond Frank Dickman and Alan Zame, Functionally compact spaces ...... 303

Ronald George Douglas and Walter Rudin, Approximation by inner

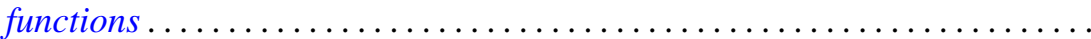

John Walter Duke, A note on the similarity of matrix and its conjugate

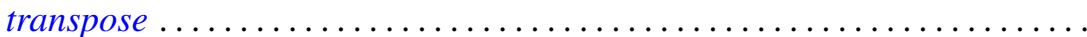

Micheal Neal Dyer and Allan John Sieradski, Coverings of mapping

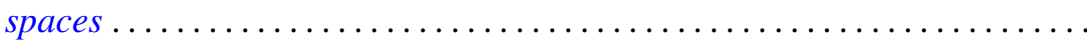

Donald Campbell Dykes, Weakly hypercentral subgroups of finite groups .....

Nancy Dykes, Mappings and realcompact spaces.....................

Edmund H. Feller and Richard Laham Gantos, Completely injective

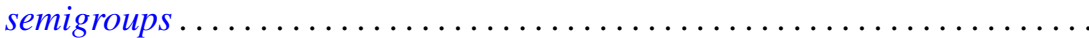

Irving Leonard Glicksberg, Semi-square-summable Fourier-Stieltjes

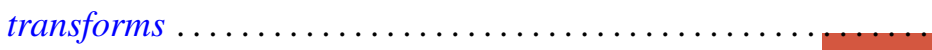

Samuel Irving Goldberg and Kentaro Yano, Integrability of almost cosymplectic structures...

Seymour Haber and Charles Freeman Osgood, On the sum $\sum\langle n \alpha\rangle^{-t}$ and numerical integration ..........................

Sav Roman Harasymiv, Dilations of rapidly decreasing functions ....

William Leonard Harkness and R. Shantaram, Convergence of a sequence of

transformations of distribution functions

Herbert Frederick Kreimer, Jr., A note on the outer Galois theory of rings ...

James Donald Kuelbs, Abstract Wiener spaces and applications to analysis. .

Roland Edwin Larson, Minimal $T_{0}$-spaces and minimal $T_{D}$-spaces...

A. Meir and Ambikeshwar Sharma, On Ilyeff's conjecture .

Isaac Namioka and Robert Ralph Phelps, Tensor products of compact convex sets....

James L. Rovnyak, On the theory of unbounded Toeplitz operators ....

Benjamin L. Schwartz, Infinite self-interchange graphs.......

George Szeto, On the Brauer splitting theorem...

Takayuki Tamura, Semigroups satisfying identity $x y=f(x$,

Kenneth Tolo, Factorizable semigroups .................. 